\title{
The influence of a ten week Tai Chi program with seniors
}

\author{
Donald N. Roberson ${ }^{1, *}$, Shu-Yi Wang ${ }^{2}$, Erik Sigmund ${ }^{1}$, and Hana Valkova ${ }^{3}$ \\ ${ }^{1}$ Faculty of Physical Culture, Palacký University Olomouc, Olomouc, Czech Republic; ${ }^{2} J o h n s$ Hopkins Center on Aging and \\ Health, Johns Hopkins University, Baltimore, MD, United States; ${ }^{3} F a c u l t y$ of Sports Studies, Masaryk University, Brno, \\ Czech Republic
}

Copyright: (C) 2015 D. N. Roberson et al. This is an open access article licensed under the Creative Commons Attribution License (http://creativecommons.org/licenses/by/4.0/).

\begin{abstract}
Background: Physical activity can help to restore physical functioning loss due to disuse and chronic illness as well as aging. Objective: There were two main aims in this study. (1) To assess the impact of a brief 10-week Tai Chi program on health-related quality of life, functional mobility, and balance performance; (2) To examine the feasibility of a ten week Tai Chi exercise program in a retirement center. Methods: Fifteen healthy voluntary elder residents (aged $76.8 \pm 9.3$ years) took place in this study. The participants were tested with the Timed Up-and-Go, One-Leg Stance, Functional Reach as well as self-reported Health Related Quality of Life (EQ-5D). The duration of this Tai Chi program was 10 weeks, with two 1 hour sessions on Monday and Wednesday mornings 9 a.m., resulting in a total of 20 hours of Tai Chi class. Results: As a result of the pre and post-test concerning the Health Related Quality of Life there was higher amount of self-care, more activities, less pain and less anxiety/depression after the ten week Tai Chi program. Comparing the pre and post-intervention Health Related Quality of Life results, significant improvements were observed for anxiety/depression and the subjective health-related quality of life scale (EQ-VAS) $(p<.05)$. Further analysis showed that the training-induced changes in Timed Up-and-Go as well as One-Leg Stance test following the intervention were significant $(p<.05)$. Conclusions: A 10-week Tai Chi program had beneficial effects on subjective health-related quality of life, functional mobility, and balance performance among the participants of this study.
\end{abstract}

Keywords: physical activity, older adults, quality of life, functional mobility, balance

\section{Introduction}

Because of the aging process and an accompanying sedentary life style, seniors often experience a decline in physical functioning and an increase in the incidence of chronic health problems (K. Chen, Hsu, Chen, \& Tseng, 2007). Aging populations are projected to cause an increase of public spending on seniors. Older adults also carry the greatest proportion of chronic disease burden, disability, and health care utilization. As a result, elderly people are becoming an increasingly important target group concerning health promotion, prevention of unnecessary functional decline, rehabilitation, and preservation of function.

Physical activity (PA) is an important goal for public health and public policy because regular PA improves well-being and contributes to the prevention or delay of

\footnotetext{
* Address for correspondence: Donald N. Roberson, Department of Social Sciences in Kinanthropology, Faculty of Physical Culture, Palacký University Olomouc, třída Míru 117, 77111 Olomouc, Czech Republic. E-mail: donald.roberson@upol.cz
}

diseases (AARP, ACSM, AGS, CDCP, \& NIA, 2001; Fried \& Freedman, 1997; Hamrik, Sigmundová, Kalman, Pavelka, \& Sigmund, 2014).

Regular PA substantially delays the onset of functional limitations and loss of independence (Gunter, White, Hayes, \& Snow, 2000). It has been reported that inactive, nonsmoking women at age 65 have 12.7 years of active life expectancy, compared with 18.4 years for highly active, nonsmoking women (Ferucci et al., 2000). Also, The American Academy of Rheumatologists recommends PA for arthritis management. One study reported that regular walking reduced pain and improved function among people with arthritis in the knees (Fransen \& McConnell, 2008). Therefore it is important that we continue to support and encourage regular PA throughout one's life.

However, PA for older adults must take into consideration the weaker status of the older adult. Many researchers have shown that Tai Chi is an appropriate exercise for older adults. Tai Chi exercises have been shown to improve strength, balance, and muscle function (Huang \& Liu, 2015; Wu, 2002; Wu, Zhao, 
Zhou, \& Wei, 2002). Tai Chi has also been shown to improve psychological functioning by reducing the fear of falling, improving self-efficacy, and overall health in the elderly (Dechamps, Onifade, Decamps, Lafont, \& Bourdel-Marchasson, 2009; F. Li, Fisher, Harmer, \& Shirai, 2003; F. Li et al., 2005; F. Li et al., 2008; F. Li et al., 2001; J. X. Li, Hong, \& Chan, 2001; Wolf, Barnhart, et al., 2003; Young, Appel, Jee, \& Miller, 1999). Tai Chi not only has beneficial effects on the components of physical condition but can produce a substantial reduction in the risk of multiple falls in older adults. Regular Tai Chi exercise has a favorable effect on the promotion of balance control, flexibility, and cardiovascular fitness in older adults. A subsequent randomized controlled trial (RCT) of Tai Chi in a similar community group demonstrated a $54 \%$ reduction in fall risk (F. Li et al., 2005).

Relevant for our study, Hartman et al. (2000) found significantly improved health satisfaction and overall arthritis self-efficacy in community-dwelling adults with lower extremity osteoarthritis who participated in a Tai Chi class totaling 24 hours. K. Chen et al. (2007) examined the effects of Tai Chi on the physical and psychological well-being of seniors who resided in long-term care facilities. Also, Tai Chi was efficient in improving Higher Quality of Life (HRQoL) of frail institutionalized elderly (Dechamps et al., 2009). Although, Tai Chi may not be as popular as it was at one time, its credibility even when compared to swimming, running, or square dancing should be extended (Zhang \& Chen, 2014).

In summary, Tai Chi has been shown to be effective in improving the lives of older adults. However, many of these exercise programs are extensive and lengthy. We wanted to find new information concerning a brief Tai Chi program. As far as we can determine this is one of the first studies of Tai Chi in the Czech Republic; further this study incorporated international focus, since the teachers of Tai Chi and the overall manager were from Taiwan. And, many of the studies of Tai Chi focus on participants from senior centers, whereas this study took place in a housing institution for the elderly.

The purpose of this research was to investigate the effects of a brief Tai Chi program with older adults on health related quality of life, functional mobility, and balance performance. Because of various time constraints, often seniors may not be willing to commit to a longer program, such as six month or even one year. Therefore, we wanted to evaluate the value of a brief Tai Chi experience. Each session was approximately one hour, with a total of 20 hours of Tai Chi instruction and practice.

\section{Methods}

\section{Participants}

This research took place at a public housing or assisted living center for seniors in Olomouc, Czech Republic. We received the written permission and consent of each person who attended this research class, as well as the permission and consent of all authorities in the retirement center. Within the retirement center there are two sections - the independent section are those seniors without frailty, whereas the dependent section includes those who need assistance. The participants were invited to attend Tai Chi from the independent section of the facility.

Initially there were 30 participants, and eventually the sample comprised 23 seniors, 5 males and 18 females, whose ages ranged from 62 to 84 (mean 74.83 , SD 6.37). However, 15 participants were able to complete the entire program. Eligibility criteria for the initial selection included: (1) to reside in the independent housing section of the retirement center; (2) to read and understand the instructions; (3) to attend the pre-intervention and post-intervention balance tests in addition to the 10-week Tai Chi program (Devereaux, Williamson, Futrell, \& Chamberlain, 1997). Individuals were excluded if they had some illness, used medicine which may impair PA, or if they had a recent surgery.

\section{Questionnaire}

The health-related quality of life was assessed according to the EQ-5D (Brooks, 1996; The EuroQoL Group, 1990). The EQ-5D is a generic self-completed health assessment instrument developed by the International EuroQol group as a standardized measure for a description of health status (Kajang, Mandy, Mark, \& Rosalind, 2009). The EQ-5D consists of two parts: the EQ-Visual Analogue Scale (EQ-VAS) is a subjective health-related quality of life scale. The second part is a descriptive questionnaire comprising five dimensions of mobility, self-care, usual activities, pain/discomfort, and anxiety/depression (Figure 1).

Respondents were asked to indicate his/her health state by rating each item on a scale from 1 (no problem) to 3 (unable, or extreme problem). The 5-digit result describes the respondents' overall health status. The EQ-5D has been shown to be applicable, reliable and valid when used with older adults in a wide variety of health-related conditions.

Eventually each member of the sample participated in three physical tests. This included the Timed Up-andGo (TUG; Podsiadlo \& Richardson, 1991), the OneLeg Stance (OLS), and the Functional Reach (FR). Before and after the intervention, each participant 


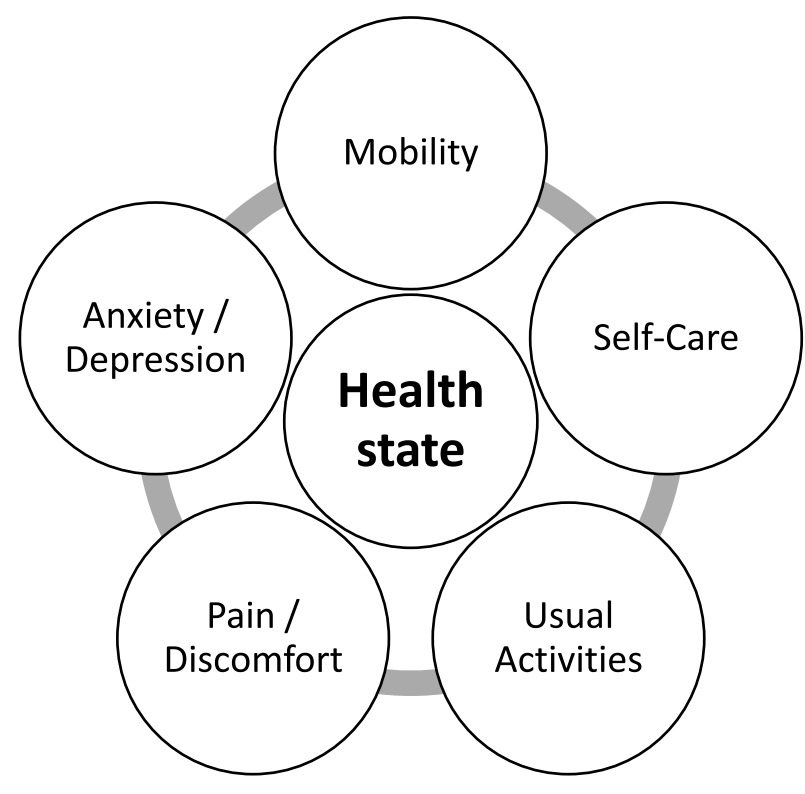

Figure 1. Composition of the health state according to EQ-5D questionnaire

performed two trials of each test, and the better score of the two trials was recorded. The protocol was kept consistent for all assessment sessions, with the same examiner for the pre and post-test.

An action plan was coordinated with the researcher and the activity coordinator for 20 Tai Chi sessions held twice a week. A recruitment announcement was made by the center and given out 10 days before the session as well as a personal invitation. With the assistance of a Czech interpreter, we gave an introduction to the nature of this Tai Chi program and presented a cultural program on Taiwan. Before the beginning of the program, the procedures were explained and the volunteers were asked to respond honestly. Then the demographic questions were asked and recorded. The EQ-5D Czech version of the questionnaires were delivered to the subjects and collected by the coordinator. Tai Chi sessions were usually conducted in the dining hall section of the center, and two sessions were outside because of appropriate weather.

\section{Procedures}

The duration of this Tai Chi program was 10 weeks, with two one-hour sessions on Monday and Wednesday mornings at 9 a.m. This resulted in a total of 20 hours of Tai Chi class. A Tai Chi instructor who is also a medical student at the local university led the group. He was assisted by a Czech interpreter who has practiced Tai Chi for more than four years. The activity coordinator of the center attended and checked the attendance during the program. Each session began with 15 minutes of warm-up, including flexibility exercises, push-hand practice in pairs, and isolated static Tai Chi movements. During the 35 minutes of core Tai Chi movement practice, the movements of each form were based on the fundamental principles of traditional Tai Chi. This includes body sensation, awareness of multidirectional weight shifting, body alignment, and multi-segmental movement coordination (F. Li et al., 2003). Deep breathing and traditional Tai Chi muscle-reinforcement exercises were integrated in the routine.

Tai chi uses a set of postures or movements. Many of these movements are named for animals or nature such as "Return to Mountain" and "Embrace Tiger". This will consist of gentle exercise and stretching performed in a slow, graceful manner. The participant is in constant motion as each posture flows into the next. All movements are coordinated with breathing. This helps to promote a calm attitude during the practice. All styles of Tai Chi focus on technique over strength. Tai Chi has an internal and an external focus on its training and brings together use of hands, arms, legs, and torso. The internal perspective is for the individual to gain control on body balance; the external is on overall condition, speed, and muscle strength. And, Tai Chi, focuses more on the internal perspective. Although Tai Chi has different styles, the overall focus is that of balance. Often two participants are working together using various "push hand" techniques. Gaining balance often means the individual must learn to change their internal center of gravity. Two participants will face each other and they will push or lunge at each other, the partner will place their hand on the other's waist or shoulders. This gentle pushing on various parts of the body enables the individual to increase their internal muscles as well as balance (C.-L. Chang, personal communication, April 24, 2012).

\section{Data analysis}

Participants who attended twelve $(60 \%)$ or fewer of twenty Tai Chi sessions were eliminated from the final data set. The data were analysed using SPSS (Version 19; SPSS, Chicago, IL). The relationship between the repeated measured variables was assessed by means of the Spearman's rank correlation coefficients. In order to define the impact of the Tai Chi program on the mobility, self-care, usual activities, pain/discomfort, anxiety/depression, and EQ-VAS a Wilcoxon Matched Pairs Test with level of significance $p<.05$ was used. EQ-5D questionnaires were coded, and the percentage of change from the pre- and post Tai Chi intervention were also calculated. 


\section{Results}

A total of 15 participants (aged $76.8 \pm 9.3$ ), twelve women and three men, were included for statistical analysis. Two out of the fifteen participants are wheelchair users with poor mobility conditions. None of them had previously practiced Tai Chi. All of the 15 participants attended at least 16 out of 20 sessions.

Concerning the Health Related Quality of Life which measures anxiety, mobility, self-care, usual activities, and pain there was a significant association between the first and second measurement due to the intervention of Tai Chi. There were moderate and significant $\left(r_{\mathrm{s}}=.54-.60, p<.05\right)$ correlations between measurements on the pre and post intervention in selfcare and pain/discomfort dimensions of EQ-5D questionnaire (Table 1).

At the end of the intervention, the sample had improved on the dimensions of mobility, pain/discomfort, and anxiety/depression as well as subjective health status. Comparing the pre and post-intervention EQ-5D results, significant improvements were observed for dimension of anxiety and depression (Table 2).

Table 1

Pre and post-intervention correlations of EQ-5D dimensions

\begin{tabular}{lll}
\hline Dimension & $r_{\mathrm{s}}$ & $p$ \\
\hline Mobility & .60 & .02 \\
Self-care & .56 & .03 \\
Usual activities & .45 & .09 \\
Pain/discomfort & .54 & .04 \\
Anxiety/depression & .47 & .08 \\
EQ-VAS & .41 & .13 \\
\hline
\end{tabular}

Note. $\quad r_{\mathrm{s}}=$ Spearman's rank correlation coefficient

Table 2

Pre and post-intervention comparison of EQ-5D dimensions

\begin{tabular}{lcl}
\hline Dimension & $T$ & $p$ \\
\hline Mobility & 0.54 & .59 \\
Self-care & 1.60 & .11 \\
Usual activities & 0.54 & .59 \\
Pain/discomfort & 0.71 & .48 \\
Anxiety/depression & 1.97 & .04 \\
EQ-VAS & 1.99 & .04 \\
\hline
\end{tabular}

Note. $T=$ Wilcoxon Matched Pairs Test.

In Figure 2 it is obvious there is a slight decrease in mobility, perhaps due to the two wheelchair users.
There is an increase in self-care, activities, a lessening of pain, and a lessening of anxiety/depression. Out of the five dimensions the two with the most improvement was self-care and less anxiety/depression. Comparing the pre and post-intervention EQ-5D results, significant improvements were observed for anxiety/ depression $(p<.05)$.

Moreover, a subjective evaluation of the personal health score (EQ VAS) was significantly higher after the intervention $(p<.05)$.

The participants had a higher score of health status $(80.73 \pm 10.91)$, they felt much better about themselves than before they were taking the Tai Chi class $(70.87 \pm 19.45)$.

In addition there were significant positive correlations in TUG $\left(r_{\mathrm{s}}=.94, p=.01\right)$ and OLS $\left(r_{\mathrm{s}}=.64\right.$, $p=.03$ ) variables shown in the Table 3 . And the next statistical test indicates there was a significant change of pre and post intervention of the Tai Chi for Timed Up-and-Go as well as One-Leg Stance.

A higher performance on TUG, OLS, and FR were indicated in Table 4. The analysis also revealed training-induced changes in TUG as well as OLS test following the intervention $(p<.05)$. The correlations of the pre-TUG $\times$ post-TUG and pre-OLS $\times$ post-OLS were significant (.94 and .64, $p<.05)$. A higher performance on TUG, OLS, and FR were indicated after the Tai Chi lessons. The analysis showed that the traininginduced changes in TUG as well as OLS test following the intervention was significant $(p<.05)$.

\section{Discussion}

The purpose of our research was to investigate the effects of a brief Tai Chi program with older adults on the health related quality of life, functional mobility, and balance performance. Our findings showed positive results with health-related quality of life and balance performance. These findings were consistent with other studies (Audette et al., 2006; Fransen, Nairn, Winstanley, Lam, \& Edmonds, 2007; F. Li et al., 2008; Wolf, Barnhart, et al., 2003; Wolf et al., 2006, Wolf, Sattin, et al., 2003). Moreover, health benefits, such as an improved self-rated physical health and advanced balance performance, were also achieved during this time. To our best knowledge, this was the first study of the effects of a Tai Chi exercise program on the healthrelated quality of life, functional mobility, and balance performance within a retirement center in the Czech Republic. Adding to this knowledge, a brief therapy is considered important because of the various time constraints of seniors as well as those who serve them. 


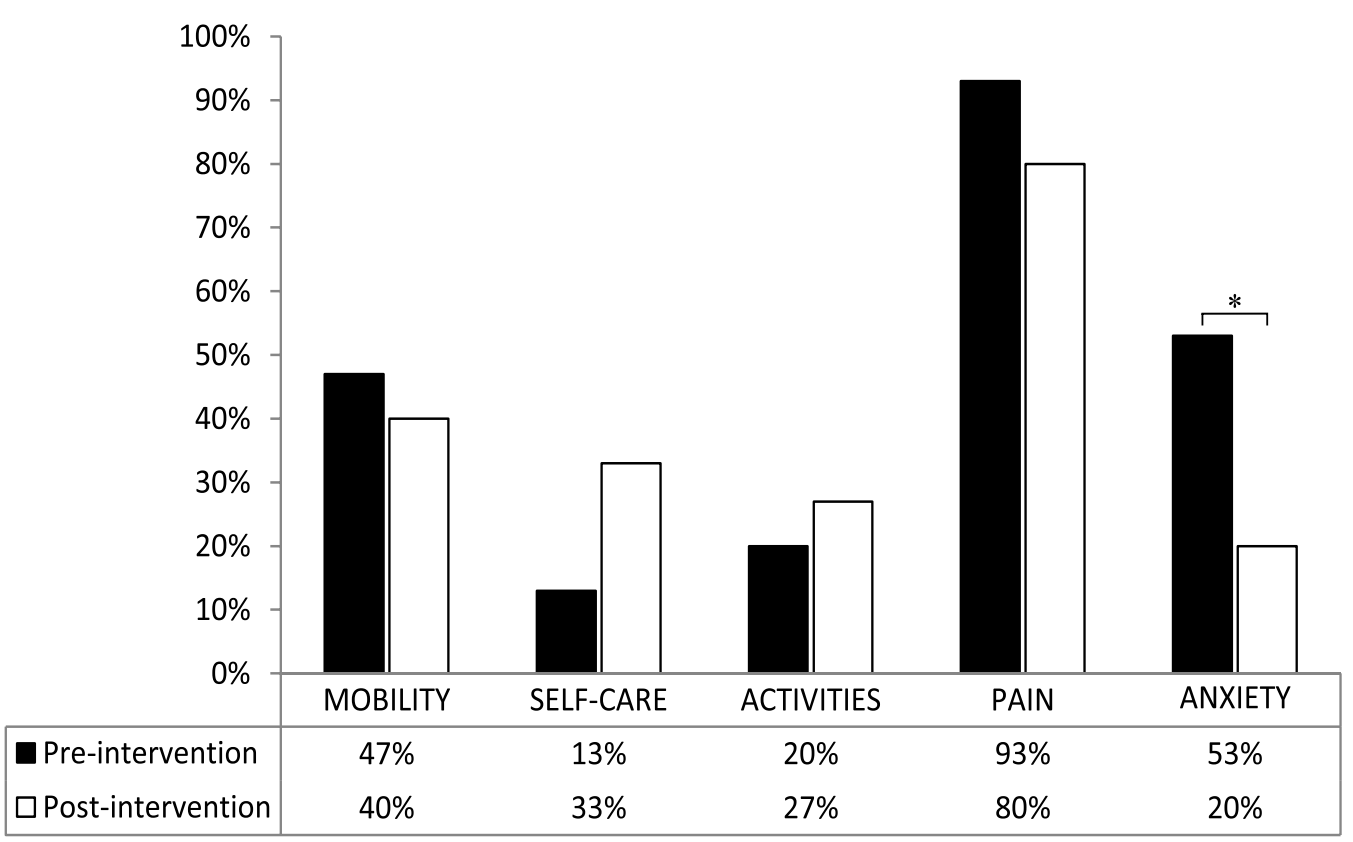

Figure 2. Percentage changes in pre and post-intervention of EQ-5D variables. ${ }^{*} p<.05$

Table 3

The correlations between pre and post-intervention of Timed Up-and-Go (TUG), One-Leg Stance (OLS) and Functional Reach (FR) test

\begin{tabular}{lll}
\hline Test & $r_{\mathrm{s}}$ & $p$ \\
\hline TUG $(\mathrm{s})$ & .94 & .01 \\
OLS $(\mathrm{s})$ & .64 & .03 \\
FR $(\mathrm{cm})$ & .17 & .60 \\
\hline
\end{tabular}

Note. $\quad r_{s}=$ Spearman's rank correlation coefficient.

Table 4

Pre and post-intervention comparison of Timed Up-and-Go (TUG), One-Leg Stance (OLS), and Functional Reach (FR) test

\begin{tabular}{lcc}
\hline Test & Pre-intervention $(M \pm S D)$ & Post-intervention $(M \pm S D)$ \\
\hline TUG (s) & $6.58 \pm 1.04$ & $6.10 \pm 0.88^{*}$ \\
OLS (s) & $15.52 \pm 21.06$ & $32.90 \pm 21.92^{*}$ \\
FR $(\mathrm{cm})$ & $77.04 \pm 5.42$ & $82.25 \pm 8.10$ \\
\hline
\end{tabular}

${ }^{*} p<.05$

This 10 week Tai Chi exercise program offered specific benefits to the participants in this study. Comparing the pre and post-intervention periods, the findings of this study also showed from our participants that: (1) less anxiety or depression was detected; (2) self-rated health score was higher; and (3) participants stated they experienced less pain or discomfort than before. These findings concur with Wang, Taylor, Pearl, and Chang (2004) whose research reported that those who participated in Tai Chi experienced greater pain reduction, less depression and improvements in physical function and overall health. Furthermore, the first finding complements previous studies that have reported a number of behavioral, social, psychological and physiological mechanisms through which mind-body interventions may lessen anxiety and depression, but few have been systematically examined in retirement centers (Bertisch, Wee, Phillips, \& McCarthy, 2009; Wang et al., 2004). Frye, Scheinthal, Kemarskaya, and Pruchno (2007) had a similar study for 12 weeks, and the Tai Chi participants also had a higher reported subjective health than others. 
The main results from our study were a better performance on TUG, OLS, and FR. However, the range of correlations with TUG and OLS was .64-.94, compared with .17 for FR (Table 4). We assert the Tai Chi exercise program improved the scores by $7 \%$ on the TUG test as well as the performance on the OLS balance test. On the other hand, FR assessing balance and stability did not improve in the same pattern.

In practical terms, activities that engage older individuals and keep their interest are needed to promote PA in this delicate age. After our introductory session, Tai Chi as a new experience seemed to capture the interest of this sample. Most participants attended at least $70 \%$ of the activity days. They were willing to help prepare the room before and after sessions. Similar to previous research, the participants also experienced the social dynamic of an exercise session $(\mathrm{K}$. Chen et al., 2007; S. Chen, Wang, \& Liu, 2006; Fransen et al., 2007). One must consider the social dynamics and atmosphere of the exercise room; having teachers from Taiwan added to the excitement of this class.

In a recent systematic review on the effects of progressive strength training, it was concluded that progressive strength training results in improvements to muscle strength and some aspects of functional limitation, such as gait speed in older adults. This results in an improved physical functioning in terms of improved balance and walking speed (Latham, Bennett, Stretton, \& Anderson, 2004). Furthermore, the effects of a general PA program in institutionalized elderly indicated a strong positive effect on muscle strength and mobility (Rydwik, Frändin, \& Akner, 2004). And, in our study, Tai Chi incorporates several movements relevant for improving physical function. While performing Tai Chi, participants are engaged in a multi-tasking PA that requires static and dynamic balance and involves turning, initiation of movement, and fluid movement patterns. The improvement in TUG after the intervention indicates that practicing Tai Chi resulted in gains in muscle strength and movement control which enhanced functional mobility. These implications were in line with previous studies that have also found a strong connection between Tai Chi exercise and functional performance (F. Li et al., 2005). There are multiple dimensions to balance requiring different measures to assess changes. Since one-leg standing is a common measure, this Tai Chi exercise program resulted in significant improvements (Audette et al., 2006; F. Li et al., 2005). Also, similar to the research of Huang and Liu (2015), our participants discussed how this class impacted their flexibility and improved their fear of falling.

Studies have shown an increasing incidence of shoulder problems as a person ages, and that the range of motion and laxity of the shoulder decreases with increasing age. Increasing loss of motion may cause an inability to perform the activities of daily living. One Tai Chi study provided an anecdotal report that one-third of the subjects experienced knee, shoulder, or lower back soreness early in the intervention (Kirsteins, Dietz, \& Hwang, 1991). Because of this, we extended the warm-up to 15 minutes and moderated the Tai Chi movements. The use of pain medication and ice packing was recommended if needed. Yet, two of our participants complained of shoulder pain.

As a result of this study we conclude that even a brief 10 week Tai Chi exercise program can improve the functional mobility and balance performance of older adults. Participation within an exercise class has additional positive effects on health-related quality of life. Viewed in this light, Tai Chi is a way for older adults to improve their health and wellness, even if they do not have time for a longer class. Adding further congruence to this research, the coordinator of the senior center as well as the program coordinator felt there were very specific impacts of having this program. The clients were interested in something new, further, having international teachers add interesting and new cultural phenomena. The participants were eager to attend and to learn this new style of exercise. This study was limited by the researchers' lack of knowledge of the Czech language. This required the assistance from an interpreter; and sometimes the translation could not convey the ideas intended. More controlled studies with larger sample sizes and outcome assessors are needed in the future to confirm and extend the present results. However, one must consider the attraction of something new, not only of Tai Chi, but also having instructors from other countries.

\section{Conclusion}

We conclude as a result of this study that even a brief Tai Chi program can promote a higher subjectiveevaluated quality of life in older adults. In addition this research confirms the previous positive results of participating in Tai Chi. Tai Chi is not just an Eastern phenomena, it is an exercise that has proved to be effective in all parts of the world and all age groups. The gentle form of movement helps to increase one's balance, lesson anxiety, and lower pain. In addition, having a class promotes a sense of community and increases available resources for the older adult. 


\section{References}

American Association of Retired People (AARP), American College of Sports Medicine (ACSM), American Geriatric Society (AGS), Centers for Disease Control and Prevention (CDCP), \& National Institute on Aging (NIA). (2001). National blueprint: Increasing physical activity among adults age 50 and older. Princeton, NJ: Robert Wood Johnson Foundation.

Audette, J. F., Jin, Y. S., Newcomer, R., Stein, L., Duncan, G., \& Frontera, W. R. (2006). Tai Chi versus brisk walking in elderly women. Age and Ageing, 35, 388-393.

Bertisch, S. M., Wee, C. C., Phillips, R. S., \& McCarthy, E. P. (2009). Alternative mind-body therapies used by adults with medical conditions. Journal of Psychosomatic Research, 66, 511-519.

Brooks, R. (1996). EuroQol: The current state of play. Health Policy, 37, 53-72.

Chen, K., Hsu, Y., Chen, W., \& Tseng, H. (2007). Well-being of institutionalized elders after Yang-style Tai Chi practice. Journal of Clinical Nursing, 16, 845-852.

Chen, S., Wang, Y., \& Liu, J. (2006). Modified stationary Tai Chi for older adults and individuals with physical disabilities. Palaestra, 22(4), 37-43.

Dechamps, A., Onifade, C., Decamps, A., \& Bourdel-Marchasson, I. (2009). Health-related quality of life in frail institutionalized elderly: Effects of a cognition-action intervention and Tai Chi. Journal of Aging and Physical Activity, 17, 236-248.

Devereaux, M. K., Williamson, E., Futrell, M., \& Chamberlain, C. (1997). A self-assessment tool to measure older adults' perceptions regarding physical fitness and exercise activity. Journal of Advanced Nursing, 25, 1220-1226.

Ferucci, L., Penninx, B. W., Leveille, S. G., Corti, M. C., Pahor, M., Wallace, R., ... Guralnik, J. M. (2000). Characteristics of nondisabled older persons who perform poorly in objective tests of lower extremity function. Journal of the American Geriatrics Society, 48, 1102-1110.

Fransen, M., \& McConnell, S. (2008). Exercise for osteoarthritis of the knee. Cochrane Database of Systematic Reviews, 4, 1-51. Retrieved from http://mrw.interscience. wiley.com/cochrane/clsysrev/articles/CD004376/ pdf_standard_fs.html

Fransen, M., Nairn, L., Winstanley, J., Lam, P., \& Edmonds, J. (2007). Physical activity for osteoarthritis management: A randomized controlled clinical trial evaluating hydrotherapy or tai chi classes. Arthritis and Rheumatism, 57, 407-414.

Fried, L., \& Freedman, M. (1997). Building communities that promote successful aging. Western Journal of Medicine, 167, 216-219.

Frye, B., Scheinthal, S., Kemarskaya, T., \& Pruchno, R. (2007). Tai chi and low impact exercise: Effects on the physical functioning and psychological well-being of older people. Journal of Applied Gerontology, 26, 433-453.

Gunter, K. B., White, K. N., Hayes, W. C., \& Snow, C. M. (2000). Functional mobility discriminates nonfallers from one-time and frequent fallers. Journals of Gerontology Series: Biological Sciences and Medical Sciences, 55, M672-M676.
Hamrik, Z., Sigmundová, D., Kalman, M., Pavelka, J., \& Sigmund, E. (2014). Physical activity and sedentary behaviour in Czech adults: Results from the GPAQ study. European Journal of Sport Science, 14, 193-198.

Hartman, C., Manos, T., Winter, C., Hartman, D., Li, B., \& Smith, J. (2000). Effects of t'ai chi training on function and quality of life indicators in older adults with osteoarthritis. Journal of the American Geriatrics Society, 48, 1553-1559.

Huang, Y., \& Liu, X. (2015). Improvement of balance control ability and flexibility in the elderly Tai Chi Chuan (TCC) practitioners: A systematic review and meta-analysis. Archives of Gerontology and Geriatrics, 60, 233-238.

Kajang, C., Mandy, O., Mark, O., \& Rosalind, R. (2009). $E Q-5 D$ user guide: Basic information on how to use EQ-5D. Rotterdam: EuroQol Group.

Kirsteins, A., Dietz, F., \& Hwang, S. (1991). Evaluating the safety and potential use of a weight-bearing exercise, tai-chi chuan, for rheumatoid arthritis patients. American Journal of Physical Medicine \& Rehabilitation, 70, 136-141.

Latham, N. K., Bennett, D. A., Stretton, C. M., \& Anderson, C. S. (2004). Systematic review of progressive resistance strength training in older adults. Journals of Gerontology Series: Biological Sciences and Medical Sciences, 59, 48-61.

Li, F., Fisher, K. J., Harmer, P., \& Shirai, M. (2003). A simpler eight-form easy tai chi for elderly adults. Journal of Aging and Physical Activity, 11, 206-218.

Li, F., Harmer, P., Fisher, K. J., McAuley, E., Chaumeton, N., Eckstrom, E., \& Wilson, N. L. (2005). Tai chi and fall reductions in older adults: A randomized controlled trial. Journals of Gerontology Series: Biological Sciences and Medical Sciences, 60, 187-194.

Li, F., Harmer, P., Mack, K. A., Sleet, D., Fisher, K. J., Kohn, M. A., ...Tompkins, Y. (2008). Tai chi: Moving for better balance - development of a community-based falls prevention program. Journal of Physical Activity and Health, 5, 445-455.

Li, F., Harmer, P., McAuley, E., Fisher, K. J., Duncan, T. E., \& Duncan, S. C. (2001). Tai chi, self-efficacy, and physical function in the elderly. Prevention Science, 2, 229-239.

Li, J. X., Hong, Y., \& Chan, K. M. (2001). Tai chi: Physiological characteristics and beneficial effects on health. British Journal of Sports Medicine, 35, 148-156.

Podsiadlo, D., \& Richardson, S. (1991). The timed 'Up \& Go': A test of basic functional mobility for frail elderly persons. Journal of the American Geriatrics Society, 39, 142-148.

Rydwik, E., Frändin, K., \& Akner, G. (2004). Effects of physical training on physical performance in institutionalised elderly patients $(70+)$ with multiple diagnoses. Age Ageing, 33, 13-23.

The EuroQol Group. (1990). EuroQol: A new facility for the measurement of health-related quality of life. Health Policy, 16, 199-208.

Wang, Y., Taylor, L., Pearl, M., \& Chang, L. (2004). Effects of Tai Chi exercise on physical and mental health of college students. American Journal of Chinese Medicine, 32, 453-459.

Wolf, S. L., Barnhart, H. X., Kutner, N. G., McNeely, E., Coogler, C., Xu, T., \& Atlanta FICSIT Group. (2003). Reducing frailty and falls in older persons: An investigation 
of tai chi and computerized balance training. Journal of the American Geriatrics Society, 51, 1794-1803.

Wolf, S. L., O’Grady, M., Easley, K. A., Guo, Y., Kressig, R. W., \& Kutner, M. (2006). The influence of intense tai chi training on physical performance and hemodynamic outcomes in transitionally frail, older adults. Journals of Gerontology Series: Biological Sciences and Medical Sciences, 61, 184-189.

Wolf, S. L., Sattin, R. W., Kutner, M., O’Grady, M., Greenspan, A. I., \& Gregor, R. J. (2003). Intense tai chi exercise training and fall occurrences in older, transitionally frail adults: A randomized, controlled trial. Journal of the American Geriatrics Society, 51, 1693-1701.

Wu, G. (2002). Evaluation of the effectiveness of Tai Chi for improving balance and preventing falls in the older population: A review. Journal of the American Geriatrics Society, 50, 746-754.

Wu, G., Zhao, F., Zhou, X., \& Wei, L. (2002). Improvement of isokinetic knee extensor strength and reduction of postural sway in the elderly from long-term Tai Chi exercise. Archives of Physical Medicine and Rehabilitation, 83, 1364-1369.

Young, D. R., Appel, L. J., Jee, S. H., \& Miller, E. R. (1999). The effects of aerobic exercise and T'ai Chi on blood pressure in older people: Results of a randomized trial. Journal of the American Geriatrics Society, 47, 277-284.

Zhang, X., Ni, X., \& Chen, P. (2014). Study about the effects of different fitness sports on cognitive function and emotion of the aged. Cell Biochemistry and Biophysics, 70, 1591-1956. 